\title{
Out-of-time-ordered correlators and quantum walks
}

\author{
Sivaprasad Omanakuttan* \\ Department of Physics and Astronomy, \\ University of New Mexico, MSC07-4220, \\ Albuquerque, New Mexico 87131-0001, USA \\ Arul Lakshminarayan $\dagger$ \\ Department of Physics, Indian Institute of Technology Madras, Chennai 600036, India
}

(Dated: April 2, 2019)

\begin{abstract}
Out-of time-ordered correlators (OTOC) have recently attracted significant attention from the physics of many-body systems, to quantum black-holes, with an exponential growth of the OTOC indicating quantum chaos. Here we consider OTOC in the context of coined discrete quantum walks, a very well studied model of quantization of classical random walks with applications to quantum algorithms. Three separate cases of operators, variously localized in the coin and walker spaces, are discussed in this context and it is found that the approximated behavior of the OTOCs is well described by simple algebraic functions in all these three cases with different time scale of growth. The quadratic increase of OTOC signals the absence of quantum chaos in these simplest forms of quantum walks.
\end{abstract}

\footnotetext{
* somanakuttan@unm.edu

$\dagger$ arul@physics.iitm.ac.in
} 


\section{INTRODUCTION}

Out-of-time-ordered correlators (OTOC) were first introduced by Larkin and Ovchinnikov in the theory of superconductivity[1]; however, it has come into prominence recently in very different contexts, finding applications in many different fields including quantum chaos, high-energy, and condensed matter physics [2 14]. It is simplest to understand it as the growth of non-commutativity of operators and hence the classical counterparts are related to Poisson brackets which can grow with time exponentially for chaotic systems. Hence it has led to discussions of the "butterfly-effect" in unconventional settings such as black holes and spin models [2, 4, 7, 15, 16]

Let the two unitary operators $A$ and $B$ be such that they commute at time $t=0$. Consider the quantity $F(t)$ directly related to the Euclidean or Frobenius norm of the commutator between the evolved operator $A(t)$ and the operator $B$, given by

$$
\begin{aligned}
F(t) & =\frac{1}{2 D}\|[A(t), B]\|_{F}^{2} \\
& =\frac{1}{2 D} \operatorname{Tr}\left([A(t), B]^{\dagger}[A(t), B]\right) \\
& =1-\frac{1}{D} \operatorname{Re}\left[\operatorname{Tr}\left(A(t)^{\dagger} B^{\dagger} A(t) B\right)\right],
\end{aligned}
$$

where $D$ is the dimension of the operators under consideration, $B(t=0)=B$ and $A(t)=$ $e^{i H t} A e^{-i H t}$ is the evolution of the operator in the Heisenberg picture (with $\hbar=1$ ). The second term in the above expression is the out-of-time-ordered correlator (other definitions need not include a full trace and may be an expectation value with respect to states such as the thermal state [7]). This governs the time evolution of the commutator, therefore define $1-F(t)$ as

$$
C_{4}(t)=\frac{1}{D} \operatorname{Re}\left[\operatorname{Tr}\left(A(t)^{\dagger} B^{\dagger} A(t) B\right)\right]
$$

If, as we assumed, the operators $A$ and $B$ commute at $t=0, F(t)$ increases from 0 in a manner that is a signature of the dynamics and can eventually saturate after a long time.

Quantum walks [17-19] come primarily in two flavors, namely the discrete and continuous time versions, and has found potential applications in quantum computation [20, 21], quantum search algorithms [22, 23] and various other fields [24 28]. The discrete quantum walk is the object of study in this paper. Due to its connections to classical random walks it is of interest to see how "chaotic" it may be with the measure of the OTOC. Independently it is an interesting dynamical question to consider. 
Discrete quantum walk consists of a walker and a coin, and its Hilbert space is a tensor product of their individual spaces. As in the classical case, the walker moves in the lattice based on the coin states. The position translation operator $\mathcal{U}$ acting on the position eigenkets $|n\rangle$ shifts them:

$$
\mathcal{U}|n\rangle=|n+1 \quad(\bmod N)\rangle
$$

where $0 \leq n<N$ and $N$ is the total number of lattice sites and we assume periodic boundary condition. Defining $\omega=\exp \left(\frac{2 \pi i}{N}\right)$, the momentum states $|\tilde{k}\rangle$ are eigenvectors of $\mathcal{U}$ :

$$
\mathcal{U}|\tilde{k}\rangle=\omega^{-k}|\tilde{k}\rangle
$$

while the momentum translation operator $\mathcal{V}$ satisfies the following relation with $l=k+$ $1 \bmod N$ :

$$
\mathcal{V}|\tilde{k}\rangle=|\tilde{l}\rangle, \quad \mathcal{V}|n\rangle=\omega^{n}|n\rangle
$$

The commutation relation between the position translation operator and the momentum translation operator is given by the standard Weyl relation [29]:

$$
\mathcal{V U}=\omega \mathcal{U} \mathcal{V}
$$

The unitary operator corresponding to the discrete quantum walk is given as [30] [18]

$$
U(\theta)=\left(|0\rangle\langle 0|\otimes \mathcal{U}+| 1\rangle\langle 1| \otimes \mathcal{U}^{\dagger}\right)\left(\tilde{U}_{\theta} \otimes \mathrm{I}_{N}\right)
$$

Here $N$ is the size of the lattice, $0 \leq \theta \leq \pi / 2$, and

$$
\tilde{U}_{\theta}=\left(\begin{array}{cc}
\cos \theta & \sin \theta \\
\sin \theta & -\cos \theta
\end{array}\right) \text {, }
$$

is the coin dynamics that occurs in a two dimensional Hilbert space spanned by the orthogonal states $\{|0\rangle,|1\rangle\}$. Due to the translational symmetry of the walk, $U(\theta)$ is block diagonal in the momentum basis of the walker $|\tilde{k}\rangle[30]$ and is given as $U(\theta)=\bigoplus_{k} U_{k}(\theta)$ where,

$$
U_{k}(\theta)=\left(\begin{array}{cc}
\cos \theta \omega^{k} & \sin \theta \omega^{k} \\
\sin \theta \omega^{-k} & -\cos \theta \omega^{-k}
\end{array}\right)
$$

and $0 \leq k<N-1$. When $\theta=\pi / 4$, this corresponds to the well-studied Hadamard coin [18]. 
There is a growing interest to understand the behavior of OTOCs where there is no quantum chaos, the recent study of OTOCs in the integrable quantum Ising model being an example. Power law growth of OTOCs are observed in integrable system as opposed to the expected exponential growth observed in nonintegrable systems exhibiting quantum chaos [31 35]. The coined discrete quantum walk is a simple model that has been posed also a a coherent version of classical random walks.

When $\theta=\pi / 4$ in the coin dynamics, measurement of the coin at each step leads to the classical unbiased random walk [18]. Thus it could be of interest to investigate how quantum chaotic this walk is. The OTOC is a measure of how two perturbations applied at different times do not commute and is a measure of the sensitivity of the system. The lack of quantum chaos in this and a phase space version of it was discussed recently in [36] and motivates one to investigate the behavior of OTOCs in various versions of quantum walks, we start therefore with the standard and simplest model in this paper, and indeed exhibit power-laws in the OTOCs, studying them both analytically and numerically.

In the discrete coined quantum walk, as mentioned above a coin and a walker space exist. In this paper a separate analysis of the OTOCs when both the operators $(A$ and $B)$ are in the coin space is carried out in the section II, similar analysis for the walker space is in section III, while in section IV we consider the case in which one operator is in the coin space and the other is in the walker space. We will conclude the paper by a summary and possible future directions in section $\mathrm{V}$.

\section{OTOCS FOR THE COIN-COIN CASE}

This section is devoted to the study of OTOC for the case in which both the operators, $A$ and $B$ as discussed in the introduction, belong to the coin space. The model of discrete quantum walk discussed in this paper has a coin with a two-dimensional Hilbert space; however there are generalizatios with higher dimensional coin spaces [37 39] which are of independent interest.

Consider $A=B=H \otimes \mathbb{1}_{N}$, which are in the coin space, where $H$ is the Hadamard gate or operator

$$
H:=\frac{1}{\sqrt{2}}\left(\begin{array}{cc}
1 & 1 \\
1 & -1
\end{array}\right)
$$


Since $H \otimes 1_{N}$ is Hermitian as well as unitary the expression of the $C_{4}(t)$ in the Eq. (2) becomes,

$$
C_{4}(t)=\frac{1}{2 N} \operatorname{Re}\left[\operatorname{Tr}\left([A(t) A]^{2}\right)\right] .
$$

However, the time evolution of the operator $A$ in the Heisenberg picture is given in terms of $U_{k}(\theta)$ as,

$$
A(t)=\sum_{k=0}^{N-1} U_{k}^{-t}(\theta) H U_{k}^{t}(\theta)
$$

which gives,

$$
F_{C C}(t)=1-\frac{1}{2 N} \sum_{k=0}^{N-1} \operatorname{Tr}\left[\left(U_{k}^{-t}(\theta) H U_{k}^{t}(\theta) H\right)^{2}\right] .
$$

The marginal cases of $\theta=0$ and $\theta=\pi / 2$ are naturally special but give rise to some interesting non-trivial behaviors for $F_{C C}$.

\section{A. Marginal Cases}

When the coin is $\sigma_{z}(\theta=0)$ and $\sigma_{x}(\theta=\pi / 2)$ further simplification of the Eq. (13) in the coin-coin case using block diagonal matrix is possible and the simplified expression of $F_{C C}(t)$ can be found, see App. (A) for details of the calculation. For the $\sigma_{z}$ coin,

$$
F_{C C}(t)=\frac{5}{4}-\frac{1}{4}\left[4(-1)^{t} \delta(2 t \bmod N, 0)+\delta(4 t \bmod N, 0)\right] .
$$

Thus $F_{C C}(0)=0$, while $F_{C C}(t \geq 1)=5 / 4$, till one of the Kronecker deltas click again which

is at time scales of the order of $N$ and is hence very long. If the initial state is $|\psi\rangle_{c}|\phi\rangle_{w}$, then the state the state after $n$ time steps is given as,

$$
|\psi(t=n)\rangle=\langle 0 \mid \psi\rangle_{c}|0\rangle \mathcal{U}^{n}|\phi\rangle_{w}+(-1)^{n}\langle 1 \mid \psi\rangle_{c}|1\rangle\left(\mathcal{U}^{\dagger}\right)^{n}|\phi\rangle_{w}
$$

Hence the initial coin state's contribution to the overall state remains same after time $t=1$, which is in-fact reflected in the behavior of $F_{C C}$.

When the coin dynamics is given by $\sigma_{x}$,

$$
F_{C C}(t)=\left\{\begin{array}{l}
0 \text { if } t \text { is even, } \\
5 / 4 \text { for } t \text { odd }
\end{array}\right.
$$

Considering the same initial state as before

$$
|\psi(t=1)\rangle=\langle 1 \mid \psi\rangle_{c}|0\rangle \mathcal{U}|\phi\rangle_{w}+\langle 0 \mid \psi\rangle_{c}|1\rangle \mathcal{U}^{\dagger}|\phi\rangle_{w}
$$


and

$$
|\psi(t=2)\rangle=|\psi(t=0)\rangle .
$$

Hence there are only two possible configurations for the system like a classical coin (no superposition of the configuration is allowed). This back and forth nature similar to the two level atom as discussed in App. (C) results in the rapid oscillation of the $F_{C C}$.

\section{B. Non-Marginal Case}

For the non-marginal cases, an alternate expression for Eq. (13) is obtained using the unitary matrix $\hat{U}=\bigotimes_{k=0}^{N-1} T_{k}$, where $T_{k}$ diagonlizes $U_{k}$, and is given as,

$$
C_{4}(t)=\frac{1}{2 N} \sum_{k=0}^{N-1} \operatorname{Tr}\left[\left(D_{k}^{-t}(\theta) A_{k} D_{k}^{t}(\theta) A_{k}\right)^{2}\right]
$$

where $A_{k}=T_{k} H T_{k}^{\dagger}$ and,

$$
D_{k}=T_{k}^{\dagger} U_{k} T_{k}=\left(\begin{array}{cc}
\exp \left(i \alpha_{k}\right) & 0 \\
0 & -\exp \left(-i \alpha_{k}\right)
\end{array}\right)
$$

with $\alpha_{k}=\sin ^{-1}(\cos \theta \sin 2 \pi k / N)$. Let

$$
A_{k}:=\left(\begin{array}{ll}
a_{11}(k) & a_{12}(k) \\
a_{21}(k) & a_{22}(k)
\end{array}\right)
$$

however, $A_{k}$ is Hermitian as well as unitary and is similar to $H$ which yields $\operatorname{Tr}\left(A_{k}\right)=0$ and $\operatorname{det}\left(A_{k}\right)=-1$, hence $a_{11}(k)=-a_{22}(k), a_{12}(k)=a_{21}(k)^{*}$, and $\left|a_{12}(k)\right|^{2}+a_{11}^{2}(k)=1$. Defining

$$
R_{k}(t):=\operatorname{Tr}\left[\left(D_{k}^{-t}(\theta) A_{k} D_{k}^{t}(\theta) A_{k}\right)^{2}\right]
$$

$\left(C_{4}(t)=\sum_{k} R_{k}(t)\right)$ can be represented in terms of $a_{11}(k)$ and $a_{12}(k)$ as,

$$
\begin{aligned}
R_{k}(t)= & 2\left[a_{11}^{4}(k)+(-1)^{t} a_{11}^{2}(k)\left|a_{12}(k)\right|^{2}\left(-2+4 \cos 2 \alpha_{k} t\right)\right] \\
& +2\left|a_{12}(k)\right|^{4} \cos 4 \alpha_{k} t
\end{aligned}
$$

Hence the expression of $C_{4}(t)$ becomes,

$$
\mathrm{C}_{4}(t)=2 \sum_{k=0}^{N-1}\left[a_{11}^{4}(k)+(-1)^{t}\left(-2+4 \cos 2 \alpha_{k} t\right) a_{11}^{2}(k)\left|a_{12}(k)\right|^{2}+\left|a_{12}(k)\right|^{4} \cos 4 \alpha_{k} t\right]
$$




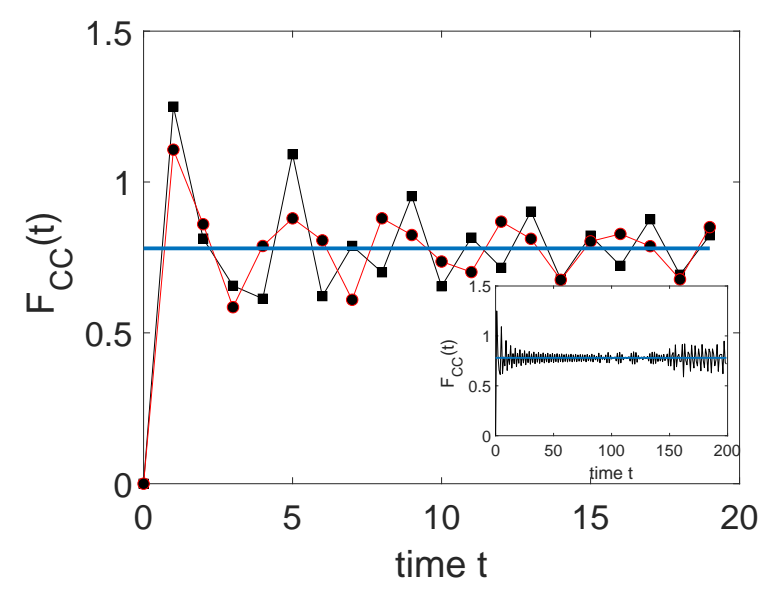

Figure 1. Growth of OTOC with time for the coin-coin case is given for $\theta=\pi / 4$, the line with square symbol is the $F_{C C}$ and the line with circle (red color) is the one obtained using the approximations, the inset gives the long time behavior of $F_{C C}(t)$ for a lattice of size $N=200$. The blue line is $y=0.78$, which shows the algebraic damping.

An exact evaluation of $a_{11}(k)$ and $a_{12}(k)$ is possible for the case of $\theta=\pi / 4$ and is given in the App. (B) . However the resultant sums appear formidable, and so we resort to using their averages over $k$ (given ahead in Eq. (B/B) ) further followed by conversion of the summation to an integral which yields the approximation

$$
\begin{aligned}
C_{4}(t) & \approx 8-\frac{11}{\sqrt{2}}+\frac{\left(1-\frac{1}{\sqrt{2}}\right)}{N} \int_{k=0}^{N} d k \cos 4 \alpha_{k} t \\
& +(-1)^{t} \frac{(-8+6 \sqrt{2})}{N} \int_{k=0}^{N} d k \cos 2 \alpha_{k} t .
\end{aligned}
$$

Since $\sin ^{-1} x \approx x$ for small values of $x$, this yields $\alpha_{k} \approx \cos \theta \sin 2 \pi k / N=1 / \sqrt{2} \sin 2 \pi k / N$, which in turn results in the final approximation of the OTOC as,

$$
\begin{aligned}
F_{C C}(t) \approx & \frac{11-7 \sqrt{2}}{\sqrt{2}}+\left(\frac{1-\sqrt{2}}{\sqrt{2}}\right) J_{0}(2 \sqrt{2} t) \\
& +(-1)^{t+1}(-8+6 \sqrt{2}) J_{0}(\sqrt{2} t) .
\end{aligned}
$$

The growth of $F_{C C}(t)$ with $t$ is given in Fig. (11), $F_{C C}(t)$ shows rapid oscillatory nature for this case in the initial time steps which tends to saturating behavior at later time. Since $J_{0}(\beta t)=J_{0}(-\beta t), F_{C C}(t)$ has time reversal symmetry. This equation shows that one relevant time scale for the coin-coin OTOC is of $O(1)$ at which it starts to oscillate. However these oscillations show an algebraic damping from the Bessel decay as $1 / \sqrt{t}$ to the constant 
value $(11-7 \sqrt{2}) / \sqrt{2} \approx 0.78$, for large $N$. The OTOC in a two level system with time independent Hamiltonian is derived in the App. (C), which shows similar behavior as the coin-coin case discussed in this section; however, it is periodic. The $F_{C C}$ in the quantum walk can be considered as a reflection of this fact that the two-level coin leads to the oscillations but due to interaction with the walker these damp out and eventually $F_{C C}$ saturates.

Since the walk analogous to the classical case happens in the lattice (walker space) and the distinction from the classical case has been the major breakthrough of the discrete quantum walk, in the next section as stated in the introduction, operators in the walker space is considered and the OTOCs is studied in detail and it is found that the OTOCs gave insights into the lack of quantum chaos in the quantum walk and also about localization of information in the walker space.

\section{OTOCS FOR THE WALKER-WALKER CASE}

When both the operators are nontrivial in the walker space, a natural choice would be when they are the momentum translation operator $\mathcal{V}$, as the complementary position translation operator $\mathcal{U}$ commutes with the quantum walk dynamics. Therefore, if we consider the operators, $A=B=\mathbb{1}_{2} \otimes \mathcal{V}$, the growth of OTOCs, $F_{W W}(t)$, in this case is an interesting

quantity. The time evolution of $A$ in the momentum basis of the walker $(|\tilde{k}\rangle)$ is given as,

$$
\left\langle\alpha \tilde{k}|A(t)| \beta \tilde{k}^{\prime}\right\rangle=\left\langle\alpha\left|U_{k}^{-t}(\theta) U_{k^{\prime}}^{t}(\theta)\right| \beta\right\rangle \delta_{k, k^{\prime}+1}^{N}
$$

Where $|\alpha\rangle,|\beta\rangle$ are arbitrary coin states and $k, k^{\prime}=0,1,2, \ldots, N-1$ gives the lattice (walker) indices. The operation of modulo on the momentum indices is represented by $\delta^{N}$. Using this, a compact formula for the OTOC is found as a sum over different momentum sectors:

$$
F_{W W}(t)=1-\frac{1}{2 N} \sum_{k=0}^{N-1} \operatorname{Tr}\left[U_{k-2}^{-t}(\theta) U_{k-1}^{t}(\theta) U_{k}^{-t}(\theta) U_{k-1}^{t}(\theta)\right] .
$$

\section{A. Marginal Cases}

The marginal cases for the walker-walker case produce trivial results; however, they provide us with some insight into the connection of OTOCs and localization. For $\theta=0$, $\left[U_{k}(0), U_{k^{\prime}}(0)\right]=0 ; \forall k, k^{\prime}$, hence quite simply $F_{W W}(t)=0$ at all times. For the case of $\theta=\pi / 2$; using Eq. (啇), results also in $U_{k-2}^{-t}(\theta) U_{k-1}^{t}(\theta) U_{k}^{-t}(\theta) U_{k-1}^{t}(\theta)=\mathbb{1}$ and therefore 
$F_{W W}(t)=0$. Thus, $F_{W W}(t)$ for both the marginal cases do not increase with time, even though the two marginal case produce two different kinds of walk on the lattice, the information encoded in them remains localized in either one or two lattice sites for all time steps. The study of participation ratio which is a measure of localization also give similar results for these marginal cases [36], and we will comment on the connections to delocalization also in the Walker-coin case.

\section{B. Non-Marginal Case}

Further simplification of $F_{W W}(t)$ in non-marginal cases is not easy. As an useful approximation, we consider the eigenvalues of $U_{k}$ to be different for different values of $k$, but we ignore the change in the eigenvectors, as $k$ changes by utmost 2 units within each product in the sum of Eq. (28). This yields,

$$
C_{4}(t)=\frac{1}{2 N} \sum_{l= \pm} \sum_{k=0}^{N-1}\left(\lambda_{k-2}^{l} \lambda_{k}^{l}\right)^{t}\left(\lambda_{k-1}^{l}\right)^{2 t}
$$

where,

$$
\lambda_{k}^{ \pm}=\exp \left( \pm i \alpha_{k}\right)
$$

are the eigenvalues of $U_{k}(\theta)$ and $\alpha_{k}$ is given by Eq. (20). This simplifies to,

$$
C_{4}(t)=\frac{1}{N} \sum_{k=0}^{N-1} \cos \left[\left(2 \alpha_{k-1}-\alpha_{k-2}-\alpha_{k}\right) t\right] .
$$

Considering that $\alpha_{k}$ change slowly for large $N$, the second difference is approximated as the second derivative

$$
\frac{d^{2} \alpha_{k}}{d k^{2}}=-\frac{4 \pi^{2}}{N^{2}} \frac{\sin \frac{2 \pi k}{N} \cos \theta \sin ^{2} \theta}{\left(1-\cos ^{2} \theta \sin ^{2} \frac{2 \pi k}{N}\right)^{3 / 2}} .
$$

Further approximating the sum as an integral yields

$$
C_{4}(t) \approx \frac{1}{2 \pi} \int_{0}^{2 \pi} \cos \left(\frac{4 \pi^{2} t \cos \theta \sin ^{2} \theta \sin x}{N^{2}\left(1-\sin ^{2} x \cos ^{2} \theta\right)^{\frac{3}{2}}}\right) d x .
$$

It is found that the numerical integration of the above is very close to the actual value $C_{4}(t)$ and is evident from the Fig. (2). Now retaining only the first two terms in the expansion of $\cos y$ and considering the case for $\theta=\pi / 4$ in the above integral yields,

$$
F_{W W}(t)=1-C_{4}(t) \approx \frac{1}{2 \sqrt{2}}\left(\frac{7 \pi^{4} t^{2}}{N^{4}}\right) .
$$



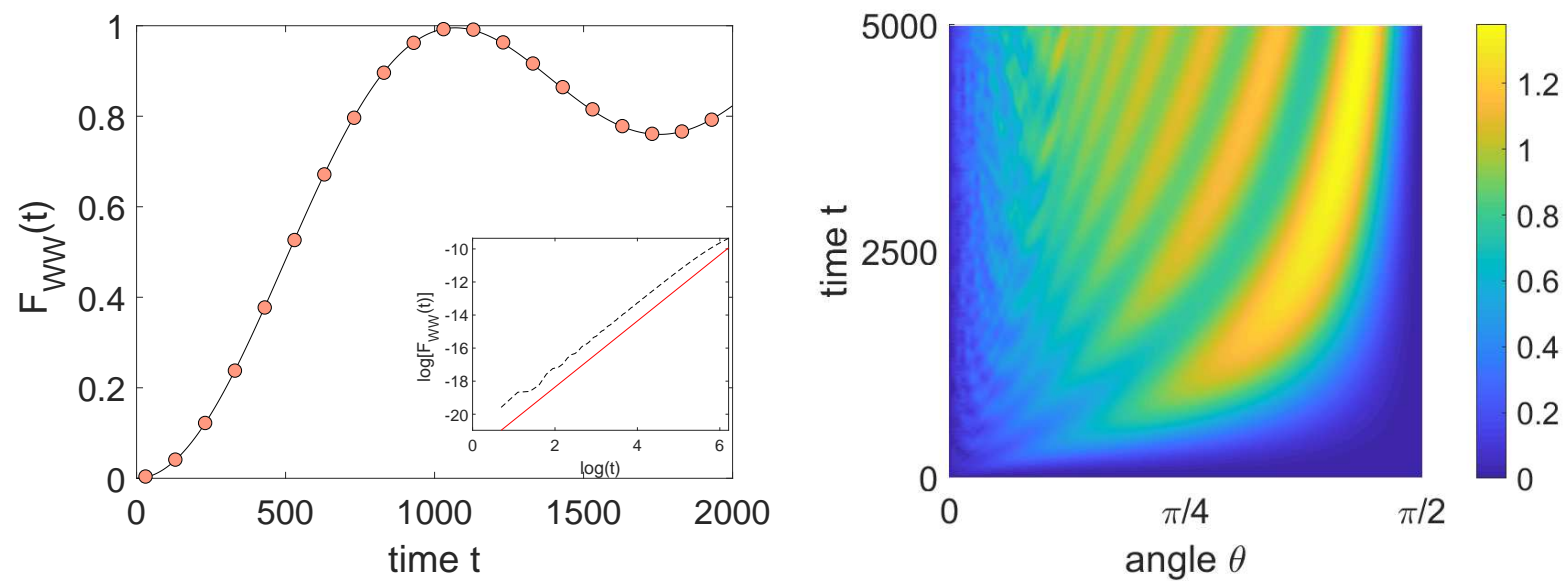

Figure 2. Growth of $F_{W W}$ with time for the walker-walker case with $\theta=\pi / 4$ is given on the left, black line is the $F_{C W}$ and the large dots are the one obtained using the numerical integration (plotted for time steps of 50), the inset figure gives the $\log$ - $\log$ graph of the same(dotted line) and the unbroken line is one with slope 2 given for comparison and the dependence of the $F_{W W}$ on time $t$ and angle $\theta$ is given on the right, for a lattice with size $N=100$.

displaying the quadratic growth phase lasting a time $\sim N^{2} / 7 \pi^{4}$. Interestingly the integral approximation is close to the actual $F_{W W}$ despite the many approximations made.

In general for the non-marginal cases the expression of $F_{W W}(t)$ is for short times approximately given as,

$$
F_{W W}(t) \approx \frac{\pi^{4} t^{2}}{2 N^{4}} \frac{\cos ^{2} \theta}{\sin \theta}(7-\cos 2 \theta)
$$

Hence the quadratic initial phase growth is valid for all values of $\theta$ except the marginal cases, up to a time scale approximately $\mathcal{O}\left(N^{2}\right)$. Also, from the above expression it is clear that growth rate is higher for $\theta$ close to zero compared to $\theta$ close to $\pi / 2$. The participation ratio, a measure of delocalization of the walker state, is correlated in the sense that it has comparitively larger value around $\theta=0$ that $\theta=\pi / 2$ [36]. The higher growth rate of $F_{W W}$ for $\theta=0$ could be considered as a consequence of this. In a somewhat different context, the OTOC also differentiates the many-body localized and thermal phases [40].

The quadratic growth of $F_{W W}(t)$ implies that the standard discrete coined model of quantum walk discussed in this paper does not exhibit any quantum chaos in the walker space. From our previous work on the growth of Eherenfest time $\left(t_{E}\right)$ with lattice dimension for discrete quantum walk $(N)$ [36], it is found that $t_{E} \sim \sqrt{N}$. However, for systems 
exhibiting quantum chaos $t_{E} \sim \log N$ and hence the algebraic growth indicates lack of quantum chaos in the system which is also substantiated by the quadratic growth of $F_{W W}$ with time.

\section{OTOCS FOR THE COIN-WALKER CASE}

The two cases considered above had operators from the same subspace, namely coin-coin or walker-walker, while OTOC is most interesting when studying cross correlations and one operator is in the coin space while the other is in the walker space. In this case OTOC indicates the scrambling of information that is localized in the coin to the walker and viceversa. Consider the operator $A=H \otimes \mathbb{1}_{N}$ in the coin space and $B=\mathbb{1}_{2} \otimes \mathcal{V}$ in the walker space. Once again using the block diagonalization of $U$ discussed in the previous sections we get the OTOC as

$$
F_{C W}(t)=1-\frac{1}{2 N} \sum_{k} \operatorname{Tr}\left[U_{k-1}^{-t}(\theta) U_{k-2}^{t}(\theta) H U_{k-1}^{-t}(\theta) U_{k}^{t}(\theta) H\right]
$$

\section{A. Marginal Cases}

For these cases further simplification of the Eq. (36) is possible and the expression of $F_{C W}(t)$ for $\theta=0$ or the $\sigma_{z}-$ coin is simply

$$
F_{C W}(t)=\sin ^{2}\left(\frac{2 \pi t}{N}\right)
$$

and for $\theta=\pi / 2$ or the $\sigma_{x}-$ coin is,

$$
F_{C W}(t)=\left\{\begin{array}{l}
0 \text { if } t \text { is even } \\
\sin ^{2}\left(\frac{2 \pi}{N}\right) \text { if } t \text { is odd }
\end{array}\right.
$$

The details of the calculation is given in the App. (D) . For $\sigma_{x}$-coin, the oscillations with time period $t=2$, is same as that of the coin-coin case counterpart given by Eq. (38); however, the amplitudes are not the same. The $\sigma_{z}$-coin shows a quadratic increase $\sim t^{2} / N^{2}$ remarkably different from the behavior of the counterpart for coin-coin, wherein $F$ attains a constant value after $t=1$ and changes very infrequently as given in Eq. (14) 


\section{B. Non marginal Cases}

In general, the four-time correlator is

$$
C_{4}(t)=\sum_{k=0}^{N-1} \operatorname{Tr}\left[U_{k-1}^{-t}(\theta) U_{k-2}^{t}(\theta) H U_{k-1}^{-t}(\theta) U_{k}^{t}(\theta) H\right]
$$

Again, further simplification of the above expression appears very hard and we need to use some approximation. Following the same routes as for the previous cases, we use the diagonal basis of the $U_{k}$, (Eq.(20) and Eq.(21)) and ignore the change in the eigenvectors for each term in the sum as $k$ changes by utmost 2. Combining these two approximations followed by some algebra yields,

$$
C_{4}(t) \approx \frac{1}{N} \sum_{k=0}^{N-1}\left[a_{11}^{2}(k) \cos \left(2 t \frac{d \alpha_{k}}{d k}\right)+\left|a_{12}(k)\right|^{2} \cos \left(t \frac{d^{2} \alpha_{k}}{d k^{2}}\right)\right]
$$

The last approximation replaced first and second differences of the phases $\alpha_{k}$ by first and second derivatives, identical to the approximation of Eq. (32) used in simplifying Eq. (31). Substituting the values of $a_{11}(k)$ and $a_{12}(k)$ from Eq. (B5) and Eq. (B6), gives the integral approximation to the above sum for the special case of the Hadamard coin, $\theta=\pi / 4$ as,

$$
\begin{aligned}
C_{4}(t) & \approx \frac{1}{2 \pi}\left[\int_{0}^{2 \pi} \frac{2 \sin ^{2} x}{3+\cos 2 x} \cos \left(\frac{\sqrt{2} \pi^{2} t \sin x}{N^{2}\left(1-\frac{\sin ^{2} x}{2}\right)^{\frac{3}{2}}}\right) d x\right] \\
& +\frac{1}{2 \pi}\left[\int_{0}^{2 \pi} \frac{4 \cos ^{2} x}{3+\cos 2 x} \cos \left(\frac{\sqrt{2} \pi t \cos x}{N\left(1-\frac{\sin ^{2} x}{2}\right)^{\frac{1}{2}}}\right) d x\right] .
\end{aligned}
$$

It is found that the numerical integration of above is close to the actual value $C_{4}(t)$ upto a time of order $\mathcal{O}(N / \pi)$ and is evident from the Fig. (3) . As

$$
F_{C W}(t) \approx \frac{0.591791}{4 \pi}\left(\frac{2 \pi t}{N}\right)^{2}
$$

the smaller time-scale indicates the time by which the coin information is scrambled and lost into the walker space. Thus this is a substantially long time and diverges as $N$ goes to $\infty$. Therefore, the growth of $F_{C W}(t)$ is quadratic, however, the time scale of growth is of $\mathcal{O}(N)$ unlike $F_{W W}$ where it is of $\mathcal{O}\left(N^{2}\right)$.

The growth of $F_{C W}(t)$ for coin-walker case is given in Fig. (3). $F_{C W}(t)$ attains its maximum value around $\theta=0$ and its minimum around $\theta=\pi / 2$. The behavior of the nonmarginal cases are almost identical except the maximum value achieved. The time scale 

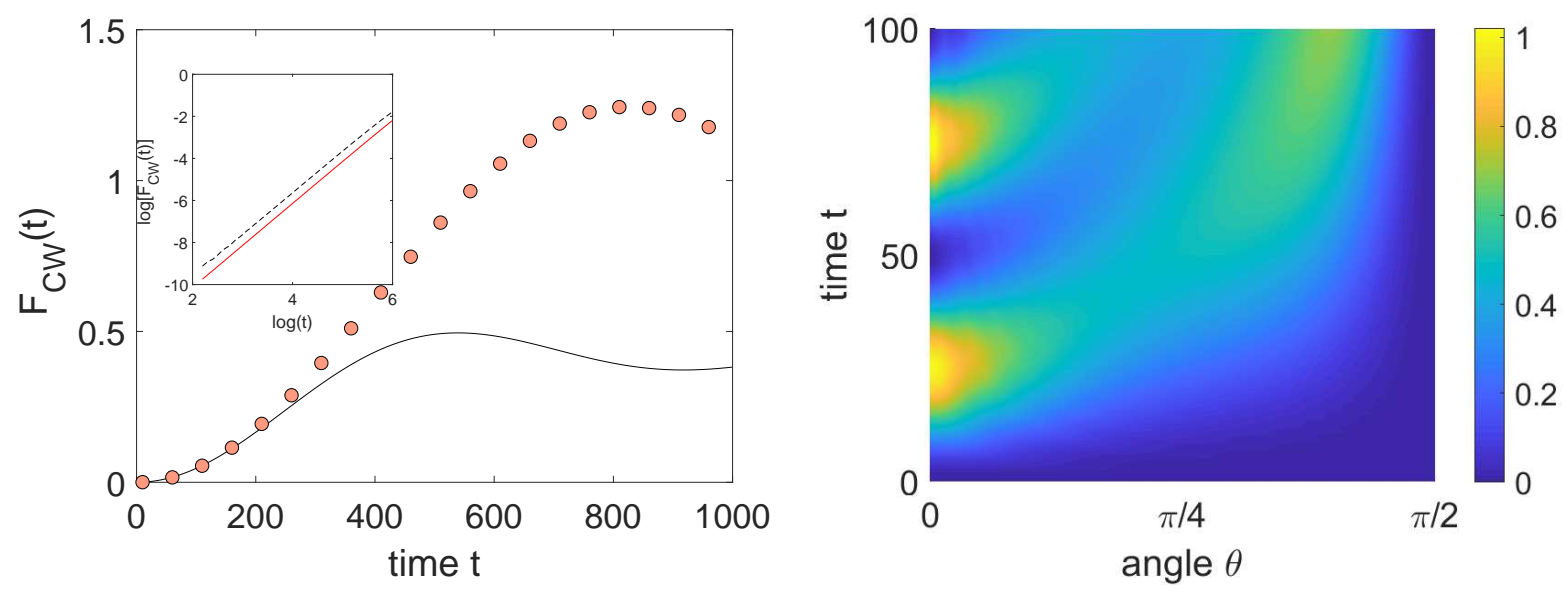

Figure 3. The growth of $F_{C W}(t)$ with time for $\theta=\pi / 4$, black line is the $F_{C W}$ and the large dots are the one obtained using the numerical integration (plotted for time steps of 50), and the inset figure gives the $\log -\log$ graph of the same (dotted line) and the unbroken line is one with slope 2 given for comparison for the coin-walker case with a lattice size $N=1000$ (left) and the dependence of $F_{C W}(t)$ on time $t$ t and angle $\theta$ (right) or a lattice of dimension $N=100$.

of growth of all these non-marginal cases are of the $\mathcal{O}(N)$. It maybe remarked that the growth of $F_{C W}(t)$ most closely resembles the growth of variance of the walker for in the coined quantum walk. Apart from the quadratic growth of both quantities, the nature of the marginal cases are similar, these cases being discussed in [36]. It may be noted that the OTOC for projector observables are closely related to transition probabilities [13] and may provide insights into connections between various dynamical quantities.

\section{SUMMARY AND CONCLUSION}

This paper studied out-of time-ordered correlators for coined discrete quantum walks with a family of coin operators parametrized by an angle. We considered separate scenarios where both operators are in the coin-space, both are in the walker-space, or where one operator belonged to each space. Since the unitary operator corresponding to the quantum walk can be block-diagonalized in the momentum basis of the walker, the OTOCs were derived largely analytically. In the case of the marginal cases of the $\sigma_{x}$-coin and $\sigma_{z}$-coin complete solutions were possible. Primarily we found that in the coin-coin case OTOC had a time scale of 
$O(1)$, beyond which it shows small fluctuations, whereas in the other two cases there was a quadrtic growth reminiscent of the variance growth in the quantum walk. Here again the most interesting case is that of the coin-walker cross OTOC which explores the scrambling between the degrees of freedom of the coin and the walker. This has a characteristic time scale of $O(N)$ and shares the maximum similarity to the variance as well. It is clear that the dimensionalities of the spaces considered determined the time-scales of the OTOC and the largest was the scrambling of the walker within the walker space, which also grows quadratically and for a time $O\left(N^{2}\right)$.

While the study reflects the lack of quantum chaos in the quantum walk, it also opens the way to analyze various other walks such as higher-dimensional cases and a complete family of coin-operators defined by $S U(2)$ coins. There is a class of quantum walk defined in [37] where the coin dynamics is governed by operators whose classical limits are chaotic, wherein the coin-coin OTOC will have an exponential growth in time. How this coin-scrambling affects the walker will be the subject of future work. We hope that our exploratory analysis of the OTOC in quantum walks enables further characterization and elucidation of the walks themselves, while at the same time add meaningfully to the burgeoning literature on the OTOC in a context not yet studied.

\section{ACKNOWLEDGMENT}

SO would like to thank Gopikrishnan Muraleedharan (CQuIC, University of New Mexico, Albuquerque) for his help during various stages of the progress of the work. 


\section{Appendix A: $F_{C C}$ for the Marginal cases}

1. Case I: $\theta=0$

$F_{C C}$ is given in terms of block diagonal matrices in the Eq. (13). Now for $\theta=0$ and $A=H \otimes \mathbb{1}$

Hence Eq. (13) becomes,

$$
U_{k}^{t}=\left\{\begin{array}{cc}
\omega^{k t} & 0 \\
0 & -\omega^{-k t}
\end{array}\right) \text { if } t \text { is odd }
$$

$$
C_{4}(t)=\frac{1}{4}\left(-1+4(-1)^{t} \sum_{k=0}^{N-1} \cos \frac{4 \pi k t}{N}+\sum_{k=0}^{N-1} \cos \frac{8 \pi k t}{N}\right)
$$

which can be further simplified as,

$$
C_{4}(t)=\frac{1}{4}\left[-1+4(-1)^{t} \delta(2 t \bmod N, 0)+\delta(4 t \bmod N, 0)\right],
$$

and thus,

$$
F_{C C}(t)=\frac{5}{4}-\frac{1}{4}\left[4(-1)^{t} \delta(2 t \bmod N, 0)+\delta(4 t \bmod N, 0)\right]
$$

2. Case II: $\theta=\frac{\pi}{2}$

For $\theta=\frac{\pi}{2}$, following some trivial algebra,

$$
U_{k}^{t}=U_{k}^{-t}=\left\{\begin{array}{c}
\left(\begin{array}{cc}
1 & 0 \\
0 & 1
\end{array}\right) \text { if } t \text { is even } \\
\left(\begin{array}{cc}
0 & \omega^{k} \\
\omega^{-k} & 0
\end{array}\right) \text { for } t \text { odd }
\end{array}\right.
$$

which in turn gives,

$$
U_{k}^{-t} H U_{k}^{t} H=\left\{\begin{array}{l}
\left(\begin{array}{cc}
1 & 0 \\
0 & 1
\end{array}\right) \text { if } t \text { is even, } \\
\frac{1}{2}\left(\begin{array}{cc}
-1+\omega^{2 k} & -1-\omega^{2 k} \\
1+\omega^{-2 k} & -1+\omega^{2 k}
\end{array}\right) \text { for } t \text { odd } .
\end{array}\right.
$$


Therefore;

$$
C_{4}(t)=\left\{\begin{array}{l}
1 \text { if } t \text { is even } \\
-\frac{1}{4} \text { for } t \text { odd }
\end{array}\right.
$$

and hence,

$$
F_{C C}(t)=\left\{\begin{array}{l}
0 \text { if } t \text { is even } \\
\frac{5}{4} \text { for } t \text { odd }
\end{array}\right.
$$

Appendix B: Explicit Calculation of $a_{11}(k)$ and $a_{12}(k)$ for $\theta=\frac{\pi}{4}$

Using Eq. (9) for $\theta=\pi / 4$ and substituting $y_{k}=\exp (2 \pi i k / N)$ yields,

$$
U_{k}\left(\frac{\pi}{4}\right)=\frac{1}{\sqrt{2}}\left(\begin{array}{cc}
y_{k} & y_{k} \\
\frac{1}{y_{k}} & -\frac{1}{y_{k}}
\end{array}\right) .
$$

The eigenvectors of which are given as:

$$
\left(\frac{1}{2}\left(1+y_{k}^{2} \pm \sqrt{1+6 y_{k}^{2}+y_{k}^{4}}\right), 1\right)
$$

which can be further simplified and $\mathcal{U}_{k}^{\dagger}$ can be written as,

$$
T_{k}^{\dagger}=\left[\begin{array}{l}
C_{-}(x)\left(\cos [x]-\sqrt{1+\cos [x]^{2}}, \exp -i x\right) \\
C_{+}(x)\left(\cos [x]+\sqrt{1+\cos [x]^{2}}, \exp -i x\right)
\end{array}\right]^{T},
$$

where $x=2 \pi k / N$ and the normalization constants $C_{ \pm}$are given as

$$
C_{ \pm}(x)=\left(2 \sqrt{\left[1+\cos ^{2} x\right]\left[1+\cos ^{2} x \pm \cos x\right]}\right)^{-\frac{1}{2}} .
$$

Now $T_{k} H T_{k}^{\dagger}$ can be calculated and following some algebra it is found that,

$$
a_{11}(k)=-\frac{2 \cos x}{\sqrt{3+\cos 2 x}},
$$

and hence,

$$
\begin{aligned}
a_{11}^{2}(k) & =\frac{4 \cos ^{2} x}{3+\cos 2 x}, \\
\left|a_{12}(k)\right|^{2} & =\frac{2 \sin ^{2} x}{3+\cos 2 x} .
\end{aligned}
$$

It is thus possible to calculate the average values,

$$
\begin{aligned}
\left\langle a_{11}^{4}(k)\right\rangle & =4-\frac{5}{\sqrt{2}}, \\
\left\langle\left|a_{12}(k)\right|^{4}\right\rangle & =1-\frac{1}{\sqrt{2}}, \\
\left\langle a_{11}^{2}(k)\left|a_{12}(k)\right|^{2}\right\rangle & =-2+\frac{3}{\sqrt{2}} .
\end{aligned}
$$




\section{Appendix C: OTOCs for the two level system}

Consider the Hamiltonian defined as,

$$
H=\cos \theta \sigma_{x}+\sin \theta \sigma_{y},
$$

and say $A=B=\sigma_{z}$, therefore

$$
A(t)=\left(\begin{array}{cc}
\cos 2 t & -i \sin 2 t e^{-i \theta} \\
i \sin 2 t e^{i \theta} & -\cos 2 t
\end{array}\right)
$$

hence $C_{4}(t)=\cos 4 t$, which gives,

$$
F(t)=1-\cos 4 t
$$

\section{Appendix D: $F_{C W}$ for the Marginal cases}

1. Case I: $\theta=0$

$F_{C W}$ is given in terms of block diagonal matrices in Eq. (36). Let $T=U_{k-1}^{-t}(\theta) U_{k-2}^{t}(\theta) H U_{k-1}^{-t}(\theta) U_{k}^{t}(\theta) H$ and using Eq. (A1) for $\theta=0$,

$$
T=\frac{1}{2}\left(\begin{array}{cc}
1+\omega^{-2 t} & 1-\omega^{-2 t} \\
-1+\omega^{-2 t} & 1+\omega^{2 t}
\end{array}\right) .
$$

which gives $C_{4}(t)=\frac{1}{2}\left(1+\cos \frac{4 \pi t}{N}\right)$, and hence

$$
F_{C W}(t)=\sin ^{2}\left(\frac{2 \pi t}{N}\right)
$$

2. Case II: $\theta=\frac{\pi}{2}$

Using Eq. (A5) for $\theta=\pi / 2$ yields,

$$
T=\left\{\begin{array}{l}
\left(\begin{array}{ll}
1 & 0 \\
0 & 1
\end{array}\right) \text { if } t \text { is even } \\
\frac{1}{2}\left(\begin{array}{cc}
1+\omega^{2} & 1-\omega^{2} \\
-1+\omega^{-2} & 1+\omega^{-2}
\end{array}\right) \text { if } t \text { is odd. }
\end{array}\right.
$$


which in turn gives,

$$
C_{4}(t)=\left\{\begin{array}{l}
1 \text { if } t \text { is even } \\
\frac{1}{2}\left(1+\cos \frac{4 \pi}{N}\right) \text { if } t \text { is odd }
\end{array}\right.
$$

therefore,

$$
F_{C W}(t)=\left\{\begin{array}{l}
0 \text { if } t \text { is even } \\
\frac{1}{2}\left(1-\cos \frac{4 \pi}{N}\right) \text { if } t \text { is odd }
\end{array}\right.
$$

[1] A. Larkin and Y. N. Ovchinnikov, Soviet Journal of Experimental and Theoretical Physics 28, 1200 (1969).

[2] J. Maldacena, S. H. Shenker, and D. Stanford, Journal of High Energy Physics 2016, 106 (2016).

[3] S. Moudgalya, T. Devakul, C. von Keyserlingk, and S. Sondhi, arXiv preprint arXiv:1808.04889 (2018).

[4] S. H. Shenker and D. Stanford, Journal of High Energy Physics 2014, 67 (2014).

[5] J. Polchinski and V. Rosenhaus, Journal of High Energy Physics 2016, 1 (2016).

[6] D. A. Roberts, D. Stanford, and L. Susskind, Journal of High Energy Physics 2015, 51 (2015).

[7] S. H. Shenker and D. Stanford, Journal of High Energy Physics 2015, 132 (2015).

[8] B. Swingle, G. Bentsen, M. Schleier-Smith, and P. Hayden, Physical Review A 94, 040302 (2016).

[9] K. Hashimoto, K. Murata, and R. Yoshii, Journal of High Energy Physics 2017, 138 (2017).

[10] S. V. Syzranov, A. V. Gorshkov, and V. Galitski, Phys. Rev. B 97, 161114 (2018).

[11] N. Tsuji, P. Werner, and M. Ueda, Phys. Rev. A 95, 011601 (2017).

[12] A. Kitaev and S. J. Suh, Journal of High Energy Physics 2018, 183 (2018).

[13] A. Lakshminarayan, Phys. Rev. E 99, 012201 (2019).

[14] A. Seshadri, V. Madhok, and A. Lakshminarayan, Phys. Rev. E 98, 052205 (2018).

[15] I. L. Aleiner, L. Faoro, and L. B. Ioffe, Annals of Physics 375, 378 (2016).

[16] D. A. Roberts and D. Stanford, arXiv preprint arXiv:1412.5123 (2014).

[17] Y. Aharonov, L. Davidovich, and N. Zagury, Physical Review A 48, 1687 (1993).

[18] J. Kempe, Contemporary Physics 44, 307 (2003).

[19] A. M. Childs, E. Farhi, and S. Gutmann, Quantum Information Processing 1, 35 (2002). 
[20] A. M. Childs, Physical review letters 102, 180501 (2009).

[21] N. B. Lovett, S. Cooper, M. Everitt, M. Trevers, and V. Kendon, Physical Review A 81, $042330(2010)$.

[22] A. M. Childs and J. Goldstone, Physical Review A 70, 022314 (2004).

[23] S. Aaronson and A. Ambainis, in Foundations of Computer Science, 2003. Proceedings. 44th Annual IEEE Symposium on (IEEE, 2003) pp. 200-209.

[24] C. Chandrashekar, R. Srikanth, and R. Laflamme, Physical Review A 77, 032326 (2008).

[25] W.-W. Zhang, S. K. Goyal, F. Gao, B. C. Sanders, and C. Simon, New Journal of Physics 18, 093025 (2016).

[26] G. Di Molfetta, M. Brachet, and F. Debbasch, Physica A: Statistical Mechanics and its Applications 397, 157 (2014).

[27] M. Genske, W. Alt, A. Steffen, A. H. Werner, R. F. Werner, D. Meschede, and A. Alberti, Physical review letters 110, 190601 (2013).

[28] G. Muraleedharan, A. Miyake, and I. H. Deutsch, arXiv preprint arXiv:1805.01858 (2018).

[29] J. Schwinger, Proceedings of the National Academy of Sciences 46, 570 (1960).

[30] A. Nayak and A. Vishwanath, arXiv preprint quant-ph/0010117 (2000).

[31] C.-J. Lin and O. I. Motrunich, Physical Review B 97, 144304 (2018).

[32] I. Kukuljan, S. Grozdanov, and T. Prosen, Physical Review B 96, 060301 (2017).

[33] J. Riddell and E. S. Sorensen, arXiv preprint arXiv:1810.00038 (2018).

[34] Y. Huang, Y.-L. Zhang, and X. Chen, Annalen der Physik 529, 1600318 (2017).

[35] Z.-H. Sun, J.-Q. Cai, Q.-C. Tang, Y. Hu, and H. Fan, arXiv preprint arXiv:1811.11191 (2018).

[36] S. Omanakuttan and A. Lakshminarayan, Journal of Physics A: Mathematical and Theoretical 51, 385306 (2

[37] A. Lakshminarayan, arXiv preprint quant-ph/0305026 (2003).

[38] S. Panahiyan and S. Fritzsche, arXiv preprint arXiv:1810.11020 (2018).

[39] L. Lorz, E. Meyer-Scott, T. Nitsche, V. Potocek, A. Gábris, S. Barkhofen, I. Jex, and C. Silberhorn, arXiv preprint arXiv:1809.00591 (2018).

[40] X. Chen, T. Zhou, D. A. Huse, and E. Fradkin, Annalen der Physik 529, 1600332 (2017). 